\title{
Tendências Genéticas para Características Relacionadas à Velocidade de Crescimento em Bovinos Nelore na Região Nordeste do Brasil ${ }^{1}$
}

\section{Carlos Henrique Mendes Malhado², Raimundo Martins Filho ${ }^{3}$, Raimundo Nonato Braga Lôbo ${ }^{4}$,

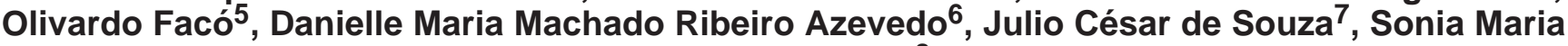 Pinheiro Oliveira ${ }^{8}$}

\begin{abstract}
RESUMO - Foram estimados os parâmetros e as tendências genéticas dos efeitos direto e materno na característica dias para ganhar $160 \mathrm{~kg}$ (D160) na fase pré-desmama e do efeito direto na característica dias para ganhar $240 \mathrm{~kg}$ (D240) na fase pós-desmama, em bovinos da raça Nelore, nascidos no período de 1965 a 2001, na região Nordeste do Brasil. Os parâmetros e as predições dos valores genéticos foram estimados utilizando o aplicativo MTDFREML. Para D160, o modelo incluiu os efeitos aleatórios genéticos direto e materno e de ambiente permanente, além dos efeitos fixos de grupo contemporâneo e da covariável idade da vaca ao parto, admitindo a covariância entre o efeito direto e materno igual a zero. Para D240 foram considerados os mesmos efeitos fixos, porém, apenas o efeito genético direto como efeito aleatório. As tendências genéticas foram estimadas pela regressão dos valores genéticos sobre o ano de nascimento dos animais. As estimativas de herdabilidade direta e materna para D160 foram, respectivamente, 0,12 $\pm 0,01$ e 0,07 $\pm 0,02$. A estimativa de herdabilidade direta para D240 foi 0,16 $\pm 0,02$. As tendências genéticas dos efeitos diretos foram significativas, com valores estimados de -0,14 e -0,41 dias/ano para D160 e D240, respectivamente. A tendência genética do efeito materno para D160 foi significativa e igual a -0,0037 dias/ano. As mudanças genéticas foram favoráveis, porém inferiores aos ganhos potenciais.
\end{abstract}

Palavras-chave: ganho genético, herdabilidade, parâmetros genéticos

\section{Genetic Trends for Traits Related to the Growth Rate in Nellore Cattle Raised in the Northeast Region of Brazil}

\begin{abstract}
The objectives of this work were to estimate the genetic parameters and the genetic trends of the direct and maternal effects for the trait days to gain $160 \mathrm{~kg}$ (D160) in the pre-weaning period, and of the direct effect for the trait days to gain $240 \mathrm{~kg}$ (D240) in the post-weaning period, of animals of Nellore breed, born from 1965 to 2001 in the Northeast region of Brazil. The analyses were carried out using the MTDFREML software. For D160 the model included the direct and maternal genetic random effects and maternal permanent environmental effect, besides the fixed effects of contemporary group and the covariate age of dam at calving, assuming the covariance between maternal and direct effect equal to zero $\left(\sigma_{\mathrm{am}}=0\right)$. For D240, the same fixed effects were considered, however, just the direct genetic effect as random. The genetic trends were estimated by linear regression of the breeding values on year of birth of the animals. The direct and maternal heritabilities for D160, respectively, were $0.12 \pm 0.01$ and $0.07 \pm 0.02$. The direct heritability for D240 was $0.16 \pm 0.02$. The genetic trends of the direct effects were significant, with estimated values of -0.14 and -0.41 days/year for D160 and D240, respectively. The genetic trend of the maternal effect for D160 was significant and equal to - 0.0037 days/year. The genetic trends were favorable, however, inferiors the potential gain.
\end{abstract}

Key Words: genetic gain, genetic parameters, heritabilities

\section{Introdução}

Contribuindo para a ocupação territorial desde o descobrimento do Brasil e para expansão da fronteira agrícola, até os dias atuais, a bovinocultura de corte destaca-se como elemento histórico do desenvolvi- mento brasileiro. Atualmente, o país possui o maior rebanho comercial do planeta, com cerca de 185 milhões de cabeças (IBGE, 2004).

Medidas de desenvolvimento ponderal, isto é, pesos e ganhos de pesos, têm servido, tradicionalmente, como critério de seleção em programas de

\footnotetext{
1 Parte da dissertação de mestrado do primeiro autor, apresentada ao programa de pós-graduação em Zootecnia da UFC. Projeto financiado pelo CNPq.

2 Doutorando em C. Biológicas, A/C Genética, Unesp/Botucatu - Professor DCB/UESB - Jequié-BA. E.mail: malhado@uesb.br

3 Pesquisador UFPI, bolsista do CNPq. E.mail: martins@ufc.br

4 Pesquisador Embrapa Caprinos. Bolsista CNPq. E.mail: lobo@cnpc.embrapa.br

5 Doutorando UFC. E.mail: ofaco@uol.com.br

6 Doutora UFC. E.mail: danizootec@bol.com.br

7 Prof DZ/UFPR. E.mail: jcs@ufpr.br

8 prof. DZ/UFC. E.mail: sonia@ufc.br
} 
melhoramento de gado de corte no Brasil (Marcondes et al., 2000). Entretanto, outros critérios de seleção, buscando velocidade e precocidade de crescimento sem aumentar o tamanho adulto dos animais, têm sido discutidos por pesquisadores e selecionadores. Entre as diversas propostas, podem ser citadas a seleção para animais pesados à idade jovem e descarte posterior de animais de maior peso adulto (Lanna, 1997) e a seleção para alterar a curva de crescimento (Fitzhugh, 1976) e dias para o animal ganhar determinado peso (Fries et al., 1996).

Considerando que o mercado possui duas unidades de comercialização definidas: o bezerro desmamado e o novilho pronto, Fries et al. (1996) propuseram a utilização das características dias para o animal ganhar $160 \mathrm{~kg}$, no período pré-desmame, e dias para ganhar $240 \mathrm{~kg}$ entre a desmama e o ponto de abate (D240), resultando na seleção sobre o tempo para atingir um produto com aproximadamente $190 \mathrm{~kg}$ na fase pré-desmame e $430 \mathrm{~kg}$ na fase pós-desmama.

Atualmente, a pecuária da região nordeste do Brasil é composta, basicamente, por animais zebuínos puros ou mestiços criados a pasto, principalmente por sua grande adaptação às condições edafoclimáticas desta região. Entretanto, os índices produtivos destes animais são baixos e têm como um dos motivos a falta de programas de melhoramento genético abrangentes.

Uma das ferramentas do melhoramento genético é a seleção e sua eficiência depende da correta identificação e utilização de animais geneticamente superiores para as características em questão. Após implantar um programa de seleção, torna-se necessário que seja periodicamente avaliado para verificar a sua eficiência (Mello, 1999).

Uma maneira de promover o monitoramento dos resultados é a avaliação do progresso genético ao longo do tempo. Como o objetivo não é só avaliar o progresso genético, mas também os resultados que sirvam de elementos orientadores de ações futuras, torna-se necessário avaliar a tendência genética ao longo do tempo (Euclides Filho et al., 1997).

No presente estudo, objetivou-se verificar se as características dias para ganhar determinado peso podem ser trabalhadas e monitorar o progresso delas por meio das estimativas das tendências genéticas dos efeitos direto e materno na característica dias para ganhar $160 \mathrm{~kg}$ na fase pré-desmama e do efeito direto na característica dias para ganhar $240 \mathrm{~kg}$ na fase pós-desmama, em bovinos da raça Nelore, nascidos no período de 1965 a 2001.

\section{Material e Métodos}

Foram utilizados dados de animais criados a pasto, nascidos no período de 1965 a 2001, no nordeste do Brasil, provenientes do controle de desenvolvimento ponderal da raça Nelore, da Associação Brasileira dos Criadores de Zebu (ABCZ).

Os cálculos de dias para ganhar $160 \mathrm{~kg}$ (D160) do nascimento ao desmame e dias para ganhar $240 \mathrm{~kg}$ (D240) na fase pós-desmama foram: D160 = $160 \mathrm{~kg}$ / GND e D240 = 240 kg/GDS, em que: GND = ganho médio diário de peso do nascimento ao desmame e GDS = ganho de peso médio diário da desmama ao sobreano.

Para a formação dos grupos contemporâneos (GC), foram consideradas quatro estações de nascimento: janeiro a março, abril a junho, julho a setembro e outubro a dezembro. Os GC continham animais do mesmo sexo, fazenda, época e ano de nascimento. Foram eliminados os registros referentes à $\mathrm{GC}$ com menos de cinco observações e os registros referentes aos bovinos que não apresentaram ganho médio diário positivo da desmama ao sobreano.

$\mathrm{Na}$ Tabela 1, consta a estrutura do conjunto de dados utilizados para D160 e D240.

Tabela 1 - Estrutura de dados no arquivo estudado para as características dias para ganhar $160 \mathrm{~kg}$ na fase pré-demama (D160) e dias para ganhar $240 \mathrm{~kg}$ na fase pós-desmama (D240), em bovinos da raça Nelore, na região nordeste do Brasil

Table 1 - Data structure in archives for the traits days to gain $160 \mathrm{~kg}$ (D160) in the pre-weaning period and days to gain $240 \mathrm{~kg}$ (D240) in the post-weaning period on Nellore cattle in Northeast Region of Brazil

\begin{tabular}{|c|c|c|}
\hline $\begin{array}{l}\text { Característica } \\
\text { Trait }\end{array}$ & D160 & D240 \\
\hline $\begin{array}{l}\text { Número total de registros } \\
\text { Total number of records }\end{array}$ & 62.002 & 22.146 \\
\hline $\begin{array}{l}\text { Número de registros utilizados } \\
\text { Number of utilized records }\end{array}$ & 46.071 & 16.226 \\
\hline $\begin{array}{l}\text { Número de touros } \\
\text { Number of sire }\end{array}$ & 2.040 & 1.013 \\
\hline $\begin{array}{l}\text { Números de vacas } \\
\text { Numberf of dam }\end{array}$ & 23.151 & 11.156 \\
\hline $\begin{array}{l}\text { Número de grupos contemporâneos } \\
\text { Number of contemporary groups }\end{array}$ & 3.529 & 1.471 \\
\hline $\begin{array}{l}\text { Média de idade da vaca ao parto (ano) } \\
\text { Mean of age of dam at calving (year) }\end{array}$ & 7,6 & 7,5 \\
\hline $\begin{array}{l}\text { Número de animais na matriz de } \\
\text { parentesco } \\
\text { Number of animals in the relationship ma }\end{array}$ & 70.031 & 27.230 \\
\hline
\end{tabular}


Para obter as estimativas das (co)variâncias e dos valores genéticos, empregou-se a metodologia da Máxima Verossimilhança Restrita Livre de Derivada (DFREML), por meio de modelos animais uni-característica, usando o aplicativo Multiple Trait Derivativ Free Restricted Maximum Likelihood (MTDFREML), desenvolvido por Boldman et al. (1995). O critério de convergência adotado foi $10^{-9}$. A cada convergência o programa era reiniciado, utilizando como valores iniciais aqueles obtidos na análise anterior até que o valor -2 $\log \mathrm{L}$ não se reduzisse mais, alcançando a convergência global.

O modelo para D160 incluiu os efeitos aleatórios genéticos, direto e materno, e de ambiente permanente, além do efeito fixo de grupo contemporâneo e da covariável idade da vaca ao parto (anos), efeitos linear e quadrático, admitindo a covariância entre os efeitos direto e materno igual a zero $\left(\sigma_{\mathrm{am}}=0\right)$.

Para D240, foram considerados os mesmos efeitos fixos, porém apenas o efeito genético direto como efeito aleatório.

As estimativas das tendências genéticas para as características D160 e D240 foram obtidas por meio da análise de regressão, utilizando-se o procedimento REG do programa SAS (1999). As variáveis dependentes foram os valores genéticos aditivos direto e materno para D160 e os valores genéticos direto para D240, enquanto a variável independente foi o ano de nascimento dos animais.

\section{Resultados e Discussão}

Na Tabela 2, encontram-se as médias, os desviospadrão e os coeficientes de variação para D160 e D240.
Considerando que o ponto ótimo seria produzir um bezerro com $190 \mathrm{~kg}$ aos 205 dias de idade e atingir o peso de abate de $450 \mathrm{~kg}$ aos 24 meses, os resultados indicam que os animais estão aquém do desejado nas duas metas, principalmente para D240. Este resultado está diretamente ligado à disponibilidade de alimentos, uma vez supõe-se que o bezerro não esteja mais mamando, deixando-o sujeito às variações na qualidade e na quantidade das pastagens.

Uma das possibilidades para reduzir o número de dias para ganhar $240 \mathrm{~kg}$ na fase pós-desmama, principalmente na estação seca, é o fornecimento de suplementação alimentar aos animais, para que não ocorra o efeito conhecido como "boi sanfona", isto é, ganho de peso nas estações chuvosas e perda no período de secas, além da seleção para animais mais adaptados à região Nordeste e com maior velocidade de crescimento.

Deve-se ressaltar que a região nordeste possui peculiaridades, decorrentes principalmente das condições edafo-climáticas, e talvez os critérios D160 e D240 não sejam os mais adequados, sendo importante definir a unidade de comercialização para a região que otimize os resultados econômicos.

A utilização do critério dias para ganhar determinado peso na seleção de gado de corte segue o exemplo das indústrias avícola e suinícola. Entretanto, deve ser considerado que, tanto em aves como em suínos, diferentemente do que ocorre em bovinos, o grau de acabamento em termos de gordura depositada na carcaça não é necessário. Portanto, nem todos os animais que ganham rapidamente os $240 \mathrm{~kg}$ na fase pós-desmama estarão prontos para o abate.

Segundo Oliveira (2003), os resultados da seleção para peso e ganhos de peso são bastante consis-

Tabela 2 - Número de observações (N), média observada, desvio-padrão e coeficiente de variação (CV) para as características dias para ganhar $160 \mathrm{~kg}$ na fase pré-desmama (D160) e dias para ganhar $240 \mathrm{~kg}$ na fase pós-desmama (D240)

Table 2 - Number of records (N), observed mean, standard deviation and coefficient of variation (CV) for the traits days to gain $160 \mathrm{~kg}(\mathrm{D160})$ in the pre-weaning period and days to gain $240 \mathrm{~kg}(\mathrm{D240})$ in the post-weaning period

\begin{tabular}{lcccc}
\hline $\begin{array}{c}\text { Característica } \\
\text { Trait }\end{array}$ & $\mathrm{N}$ & $\begin{array}{c}\text { Média (dias) } \\
\text { Mean (days) }\end{array}$ & $\begin{array}{c}\text { Desvio-padrão } \\
\text { Standard deviation }\end{array}$ & CV (\%) \\
\hline D160 & 46.071 & 265,2 & 58,6 & 22,1 \\
D240 & 16.226 & 718,4 & 244,6 & 34,0 \\
\hline
\end{tabular}


tentes em relação à teoria, enquanto dias para ganhar determinado peso, proposto por Fries et al. (1996), nunca foi testado em um experimento de longa duração.

$\mathrm{Na}$ Tabela 3, encontram-se os componentes de (co)variâncias e as herdabilidades direta e materna estimadas para as duas características. A estimativa de herdabilidade direta para D160 foi $0,12 \pm 0,01$, valor muito próximo ao obtido por Muniz (2001). Resultados superiores foram relatados por Peña (1998), Garnero et al. (2001), Marcondes et al. (2000), Simonelli et al. (2001) e Oliveira (2003), com valores que variaram de 0,14 a 0,23 para animais da raça Nelore. Entretanto, Barros et al. (2003) relataram herdabilidade de 0,10 para animais da raça Guzerá.

A estimativa de herdabilidade materna para D160 foi $0,07 \pm 0,02$, indicando a importância do efeito da mãe nesta fase de vida do animal. Marcondes et al. (2000) observaram herdabilidade materna igual a zero, contudo, diversos autores relataram herdabilidades maternas para zebuínos, com valores variando de 0,05 a 0,16 (Albuquerque \& Fries, 1998; Peña, 1998; Garnero et al., 2001; Simonelli et al., 2001; Souza et al., 2002; Barros et al., 2003; Oliveira, 2003).

A estimativa da herdabilidade direta para D240 foi $0,16 \pm 0,02$, superior àquela descrita por Souza et al. (2002) e Barros et al. (2003), ambos trabalhando com a raça Guzerá, e inferior aos relatados por Peña (1998) e Oliveira (2003) para a raça Nelore.

As estimativas de herdabilidade direta relatadas para D160 e D240, neste estudo, indicam possibilidade de ganhos genéticos por meio da seleção. Embora a estimativa da herdabilidade materna seja de menor magnitude, quando comparada com a herdabilidade direta, progressos genéticos podem ser obtidos caso haja seleção sobre a herança materna.

$\mathrm{O}$ efeito de ambiente permanente para a característica D160 foi alto $(0,14)$, o que indica diferenças no ambiente proporcionado pela mãe aos seus diferentes filhos. Este efeito pode ser decorrente de incidentes que afetam a produção de uma mesma vaca, como, por exemplo, ocorrência de mastite, acidentes no úbere ou sequielas de alguma doença. Contudo, deve ser lembrado que os efeitos maternos genéticos não aditivos também estão incluídos nesta estimativa.

As tendências genéticas para os efeitos diretos para as características D160 e D240 foram significativas $(\mathrm{P}<0,001)$ e iguais a $-0,14$ e $-0,41$ dias/ano. Em termos de mudança genética anual, isto representa 0,053 e $0,055 \%$, com ganhos genéticos nos 36 anos de estudo de 5,04 e 14,76 dias a menos para ganhar 160 e $240 \mathrm{~kg}$, respectivamente (Figuras 1 e 2). Souza et al. (2002) verificaram para a raça Guzerá, tendências de $-0,20$ e -0,68 dias/ano para D160 e D240, enquanto Muniz (2001) observou ganho de -0,105 dias/ano para D160 na raça Gir.

A tendência genética materna foi significativa $(\mathrm{P}<0,05)$ e igual a $-0,0037$ dias/ano para D160, com um ganho total nos 36 anos de 0,133 dias a menos para ganhar $160 \mathrm{~kg}$ (Figura 3), resultando numa contribuição quase nula, o que reflete a inexistência de seleção materna para esta característica. Resultados indicando valores muito pequenos foram relatados por Muniz (2001) e Souza et al. (2002), para este efeito, nas raças Gir e Guzerá, respectivamente.

Na Figura 1, pode-se observar que até o ano de 1987, a tendência genética do efeito direto para D160

Tabela 3 - Estimativas dos componentes de (co)variância e herdabilidades para as características dias para ganhar $160 \mathrm{~kg}$ na fase pré-demama (D160) e dias para ganhar $240 \mathrm{~kg}$ na fase pós-desmama (D240)

Table 3 - Estimates of (co)variance components and heritabilities for the traits days to gain $160 \mathrm{~kg}$ (D160) in the pre-weaning period and days to gain $240 \mathrm{~kg}$ (D240) in the post-weaning period

\begin{tabular}{|c|c|c|c|c|c|c|c|}
\hline & $\begin{array}{c}\sigma^{2} \mathrm{a} \\
\left(\operatorname{dias}^{2}\right) \\
d^{2} a y s^{2}\end{array}$ & $\begin{array}{c}\sigma^{2} \mathrm{~m} \\
\left(\operatorname{dias}^{2}\right) \\
\text { days }^{2}\end{array}$ & $\begin{array}{c}\sigma_{\text {ep }}^{2} \\
\left(\text { dias }^{2}\right) \\
\text { days }^{2}\end{array}$ & $\begin{array}{c}\sigma^{2} \mathrm{e} \\
\left(\operatorname{dias}^{2}\right) \\
d a y s^{2}\end{array}$ & $\begin{array}{c}\sigma^{2} \mathrm{p} \\
\left(\operatorname{dias}^{2}\right) \\
\text { days }^{2}\end{array}$ & $\mathrm{~h}_{\mathrm{d}}^{2}$ & $\mathrm{~h}_{\mathrm{m}}^{2}$ \\
\hline D160 & 210,9 & 127,7 & 251,9 & 1186,0 & 1776,6 & 0,12 & 0,07 \\
\hline D240 & 4057,8 & - & - & 21303,4 & 25361,2 & 0,16 & - \\
\hline
\end{tabular}

$\sigma^{2}{ }_{\mathrm{a}} ; \sigma^{2}{ }_{\mathrm{m}} ; \sigma^{2}{ }_{\mathrm{ep}} ; \sigma^{2}{ }_{\mathrm{e}} ; \sigma^{2}{ }_{\mathrm{p}} ; \mathrm{h}^{2}{ }_{\mathrm{d}} ; \mathrm{h}_{\mathrm{m}}^{2}=$ componentes de variância genética aditiva direta, genética aditiva materna, variância de ambiente permanente, variância residual, variância fenotípica, herdabilidade direta e materna, respectivamente.

$\sigma^{2}{ }_{a} ; \sigma^{2}{ }_{m} ; \sigma^{2}{ }_{e p} ; \sigma^{2}{ }_{e} ; \sigma^{2}{ }_{p} ; h^{2} ; h^{2}{ }_{m}=$ components of direct additive genetic, maternal additive, permanet environmental variance, residual variance, direct heritability and maternal heritability, respectively 


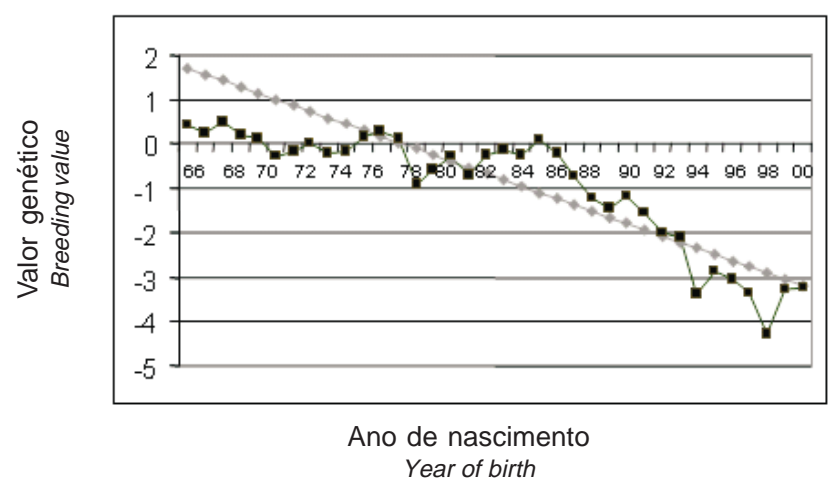

Figura 1 - Tendência genética (dias) do efeito direto para D160 no período de 1965 a 2001.

Figure 1 - Genetic trend (days) of the direct effect for D160 from 1965 to 2001.

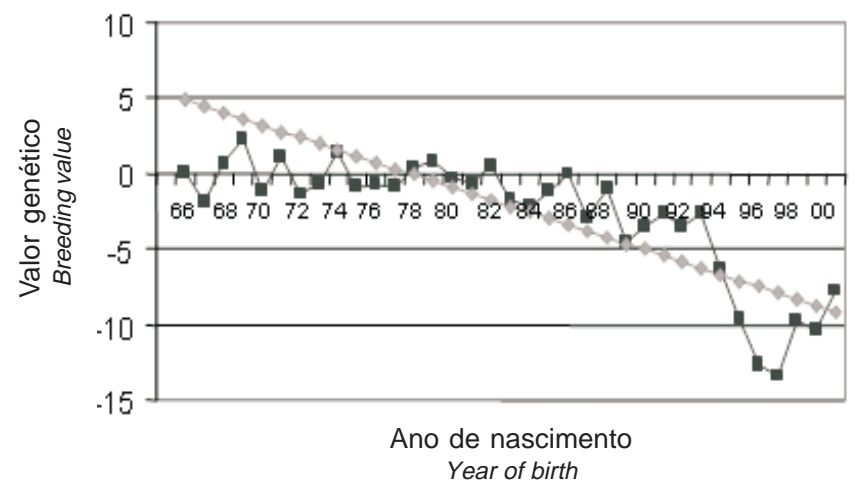

Figura 2 - Tendência genética (dias) do efeito direto para D240 no período de 1965 a 2001.

Figure 2 - Genetic trend (days) of the direct effect for D240 from 1965 to 2001.

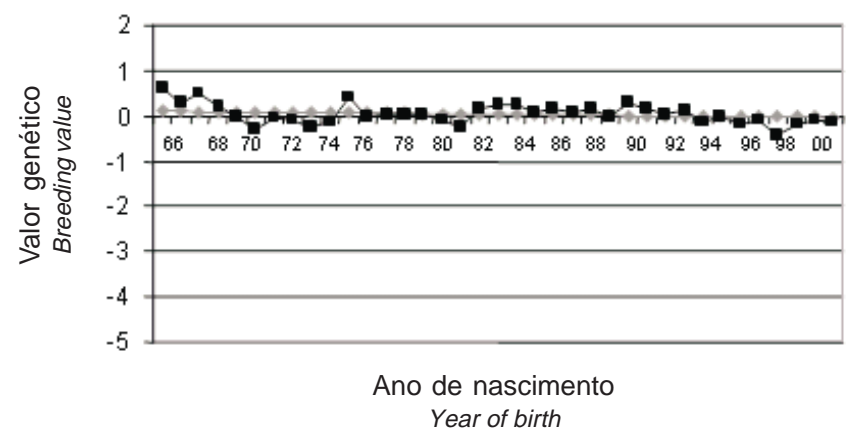

Figura 3 - Tendência genética (dias) do efeito materno para D160 no período de 1965 a 2001.

Figure 3 - Genetic trend (days) of the maternal effect for D160 from 1965 to 2001. manteve-se praticamente estável, sendo de -0,05 dia/ ano o ganho genético no período de 1987 a 2001, o que representa $62,1 \%$ do ganho genético do período total estudado (36 anos). Para D240, o ganho genético no período de 1993 a 2001 foi de $-5,0$ dias/ano, representando $35 \%$ do ganho genético de todo o período (Figura 2), indicando a existência de seleção nos últimos anos para as características relacionadas à velocidade de crescimento, em alguns rebanhos da região. Entretanto, deve ser ressaltado que os ganhos genéticos observados para as características deste estudo provavelmente ocorreram de forma indireta, por resposta correlacionada a partir da seleção para pesos corporais e ganhos de peso, visto que as características dias para atingir determinado peso não são critérios de seleção utilizados na maioria destes rebanhos.

Segundo Silva et al. (2002), usualmente as mudanças fenotípicas nas características reprodutivas e produtivas têm no ambiente sua maior contribuição.

Considerando-se intensidade de seleção de 1,46, isto é, a retenção de $2 \%$ dos machos e $70 \%$ das fêmeas, as herdabilidades direta da Tabela $3(0,12$ e $0,16)$, os desvios padrão fenotípicos da Tabela $2(58,6$ e 244,6 dias) e assumindo um intervalo de geração de cinco anos, seria possível obter progressos genéticos ("G) por ano da ordem de 2,05 e 11,4 dias/ano, para D160 e D240, respectivamente, correspondendo a 0,77 e $1,58 \%$ da média da população. De acordo com Smith (1985), a taxa de mudança genética anual é de 1 a $3 \%$, destacando os modestos ganhos genéticos obtidos para os efeitos diretos observados neste estudo.

Segundo Biffani et al. (1999), nas condições de criação típicas do nordeste, os criadores selecionam os animais mais pelas características anatômicas e raciais que pelo desempenho produtivo. Entretanto, esta tendência vem diminuindo nos últimos anos, conforme podemos verificar neste trabalho. Portanto, a população estudada pode alcançar maiores ganhos genéticos que permitam a alteração das médias para D160 e D240, refletindo em maior velocidade de crescimento dos animais.

\section{Conclusões}

Os animais requereram muitos dias para ganhar $240 \mathrm{~kg}$ no período pós-desmama, o que indica que ações devem ser implementadas para que estes possuam melhor desenvolvimento neste período. 
As mudanças genéticas para o efeito direto foram favoráveis, refletindo em menor número de dias para D160 e D240, porém inferiores aos ganhos potenciais. O baixo valor de ganho genético decorrente do efeito materno na característica D160 indica a inexistência de seleção para o efeito materno.

\section{Literatura Citada}

ALBUQUERQUE, L.G; FRIES L.A. Selection for reducing ages of marketing units in beef cattle. In: WORLD CONGRESS ON GENETICS APPLIED TO LIVESTOCK PRODUCTION, 6., 1998, Armidale Proceedings... Austrália: 6WCGALP, 1998. v.27, p.235-238.

BARROS, C.S.; MYAGUI, A.P.; SOUZA, J.C. et al. Estimativas de parâmetros genéticos sobre dias para ganhar 160 e $240 \mathrm{~kg}$ nas fases pré e pós desmamem de bovinos guzerá em duas regiões paulistas. In: REUNIÃO ANUAL DA SOCIEDADE BRASILEIRA DE ZOOTECNIA, 40., 2003, Santa Maria. Anais... Santa Maria: Sociedade Brasileira de Zootecnia, 2003, CD-ROM.

BIFFANI, S.; MARTINS FILHO, R.; GIORGETTI, A. et al. Fatores ambientais e genéticos sobre o crescimento ao ano e ao sobreano de bovinos Nelore, criados no Nordeste do Brasil. Revista Brasileira de Zootecnia, v.28., n.3, p.468-473, 1999.

BOLDMAN, K.G.; KRIESE, L.A.; Van VLECK, L.D. et al. A manual for use of MTDFREML: a set of programs to obtain estimates of variances and covariances [DRAFT]. Lincoln: Department of Agriculture, Agricultural Research Service, 1993. 120p.

EUCLIDES FILHO, K.; NOBRE, P.R.C.; ROSA, A.N. Tendência genética na raça Guzerá. In: REUNIÃO ANUAL DA SOCIEDADE BRASILEIRA DE ZOOTECNIA, 34., 1997, Juiz de Fora. Anais... Juiz de Fora: Sociedade Brasileira de Zootecnia, 1997. p.175.

FITZHUGH, JR.H.A. Analysis of growth curves and strategies for altering their shape. Journal of Animal Science, v.42, n.4, p.1036-1051, 1976.

FRIES, L.A; BRITO, F.V.; ALBUQUERQUE, L.G. Possíveis conseqüências de seleção para incrementar pesos às idadespadrão vs. reduzir idades para produzir unidades de mercado. In: REUNIÃO ANUAL DA SOCIEDADE BRASILEIRA DE ZOOTECNIA, 33., 1996, Fortaleza. Anais... Fortaleza: Sociedade Brasileira de Zootecnia, 1996, p.310-312.

GARNERO, A.V.; LÔBO, R.B.; BEZERRA, L.A.F.; OLIVEIRA, H.N. Comparação entre alguns critérios de seleção para crescimento na raça Nelore. Revista Brasileira de Zootecnia, v.30, n.3, p.714-718, 2001.

IBGE, 2004. Disponível em http://www.sidra.ibge.gov.br/bda/ pecua/default.asp Acesso em: 09/08/2004.
LANNA, D.P. Fatores condicionantes e predisponentes da puberdade e da idade ao abate. In: SIMPÓSIO SOBRE PECUÁRIA DE CORTE, 4., 1996, Piracicaba. Anais... Piracicaba: Fundação de Estudos Agrários Luiz de Queiroz, 1997. p.41-78.

MARCONDES, C.R.; BERGMANN, J.A.; ELER, J.P. et al. Análise de alguns critérios de seleção para características de crescimento na raça Nelore. Arquivo Brasileiro Medicina Veterinária e Zootecnia, v.52, n.1, p.83-89, 2000.

MELLO, S.P. Tendência genética para pesos em um rebanho da raça Canchim. Jaboticabal: Universidade Estadual Paulista, 1999. 78p. Dissertação (Mestre em Zootecnia) Universidade Estadual Paulista, 1999.

MUNIZ, C.A.S.D. Estudo de dois critérios de seleção na prédesmama de bovinos da raça Gir. Jaboticabal: Universidade Estadual Paulista 2001. 130 p. Tese (Doutorado em Zootecnia). Universidade Estadual Paulista, 2001.

OLIVEIRA, H.N. Comparação de critértios de seleção em gado de corte. Botucatu: Universidade Estadual Paulista, 2003. 133p. Tese (Livre-Docência) - Universidade Estadual Paulista, 2003.

PEÑA, C.D.O. Análise de critérios de seleção para precocidade sexual e de crescimento de bovinos da raça Nelore, no Paraguai. Jaboticabal: Universidade Estadual Paulista, 1998. 143p. Dissertação (Mestrado em Zootecnia) Universidade Estadual Paulista, 1998.

STATISTICAL ANALYSIS SYSTEM - SAS. User's guide. Cary: 1999.

SIMONELLI, S.M.; SILVA, M.A.; SILVA, L.O.C. et al. Critérios de seleção para características de crescimento no período prédesmame em bovinos da raça Nelore. In: REUNIÃO ANUAL DA SOCIEDADE BRASILEIRA DEZOOTECNIA, 38., 2001., Piracicaba. Anais... Piracicaba: Sociedade Brasileira de Zootecnia, 2001. p.675-676.

SILVA, L.O.C.; ROSA, A.N.; GONDO, A. et al. Estudo de tendências genéticas de características produtivas e reprodutivas na raça Tabapuã. In: REUNIÃO ANUAL DA SOCIEDADE BRASILEIRA DE ZOOTECNIA, 39., 2002, Recife. Anais... Recife: Sociedade Brasileira de Zootecnia, 2002. CD-ROM.

SMITH, C. Rates of genetic change in farm livestock. Research Development Agricultural, v.1, n.2, p.79-85, 1985.

SOUZA, J.C.; MALHADO, C.H.M.; SILVA, L.O.C. et al. Causas de variação e tendência genética para dias para ganhar 160 e $240 \mathrm{~kg}$ em bovinos Guzerá criados na região Nordeste.In: SIMPÓSIO NACIONAL DE MELHORAMENTO ANIMAL, 4., 2002, Campo Grande. Anais... Campo Grande: Sociedade Brasileira de Melhoramento Animal, 2002. p.173-175.

Recebido em: 04/02/04 Aceito em: 21/10/04 